\section{Aus der Geschäftsstelle}

\section{swissinformatics.org}

Die Web Site (www.s-i.ch) wird bis Ende 2016 durch ein Online Magazine auf swissinformatics.org ersetzt. Zudem wird die neue WebSite durch Subdomains ergänzt und diese werden ausserdem weitere SIPublikationen wie den Newsletter (news.swissinformatics.org) und das SISR Magazine (sisr.swiss informatics.org) umfassen. In Planung sind weitere Subdomains wie education.swissinformatics.org, social-responsibility.swiss informatics.org sowie Webauf- tritte von Fachgruppen. Für Fragen wenden Sie sich bitte an office(at)swissinformatics.org.

\section{Schweizer Informatik Gesellschaft: Studie zur Digitalen Transformation in Industrie und Gesellschaft}

\section{November 2016, \\ 8:00-12:00 Uhr}

Die Schweizer Informatik Gesellschaft führt im Rahmen ihrer strategischen Aktivitäten im Gebiet „Informatik und Gesellschaft" eine Studie zum Thema „Digitale Transformation in Industrie und Gesellschaft" durch. Im Fokus stehen Fragen rund um das Bewusstsein dieser Transformation, den
Stand aktueller Implementationen, die Nachhaltigkeit des wirtschaftlichen und politischen Umfeldes und die antizipierten Konsequenzen dieser Entwicklung.

Weitere Informationen und Umfrage: http://sgiz.mobi/s3/ Digital-Transformation-and-Society

\section{Veranstaltungen}

Eine Übersicht über Veranstaltungen finden Sie bei https://news.swiss informatics.org/de/?tribe_events_cat= veranstaltungen.

Weitere Veranstaltungshinweise: http://www.s-i.ch/veranstaltungen/ 